\title{
DIVISION IV / COMMISSION 26 / WORKING GROUP BINARY AND MULTIPLE SYSTEM NOMENCLATURE
}

\author{
CO-CHAIRS \\ MEMBERS
}

\author{
Brian D. Mason, William I. Hartkopf \\ Dimitri Pourbaix, Colin D. Scarfe, \\ Marion Schmitz, Andrei A. Tokovinin
}

\section{TRIENNIAL REPORT 2006 - 2009}

\section{Introduction}

The Working Group on Binary and Multiple System Nomenclature was formed within Commission 26 following Special Session 3 held during the 2003 Sydney General Assembly. Its purpose is to create the Washington Multiplicity Catalog, a comprehensive database first introduced at a multi-commission meeting at the IAU XXIV General Assembly in Manchester, 2000. Data are being compiled from the US Naval Observatory visual binary catalogs and supplemented with binary and multiple star information from other sources to include but not limited to spectroscopy, photometry, eclipsing and interacting system, as well as extra-solar planets and substellar companions. The goal being creation of a comprehensive hierarchical database and to reduce confusion from multiple nomenclature schemes used by disparate techniques.

\section{Activities}

Following the Manchester Multi-Commission Meeting, a sample slice of the sky was selected for implementation. This sample, of 30' width in Right Ascension and from poleto-pole was completed for the Sydney Special Session. Of this portion of the sky, various techniques contribute to the sample WMC in the following percentages:

$95.8 \%$ visual binaries and optical pairs

$50.6 \%$ interferometric binaries and optical pairs

$1.7 \%$ spectroscopic binaries

$1.4 \%$ cataclysmic variables or related objects

$1.0 \%$ occultation binaries

$0.3 \%$ astrometric binaries

$0.2 \%$ eclipsing binaries

$0.2 \%$ X-ray binaries

$0.1 \%$ spectrum binaries

$0.1 \%$ planets

Since the techniques are complementary, the sum is $>100 \%$. It should be noted that this breakdown is biased significantly by selection effects. For example, while visual binaries may be discovered (and cataloged) after a single observation, data on spectroscopic pairs are often not published until the full orbit has been characterized.

To identify common objects, precise $\left(0^{\prime \prime} .1\right)$ coordinates were selected to form the sieve by which objects are brought into multiplicity arrangements. While the more modern 
techniques often list precise coordinates as a matter of course, the older and much larger visual database does not. To date, work has focused on completing this. To date, the completion status is as follows:

Status of WDS coordinates

Supplementary information

$\begin{array}{rll}0.1 \text { arcsec level } & 98990 & 95.34 \% \\ 1 \text { arcsec level } & 993 & 0.96 \% \\ 10 \text { arcsec level } & 1284 & 1.24 \% \\ \text { worse than 10 arcsec } & 1804 & 1.74 \% \\ \text { rejected (bogus) } & 753 & 0.73 \%\end{array}$

two proper motions $4211240.56 \%$

one proper motion $4350141.90 \%$

no proper motion $18211 \quad 17.54 \%$

no primary magnitude $54 \quad 0.05 \%$

no secondary magnitude $2128 \quad 2.05 \%$

$\begin{array}{rlr}\text { no theta } & 228 & 0.22 \% \\ \text { no rho } & 36 & 0.03 \% \\ \text { no observation date } 28 & 0.03 \%\end{array}$

\section{Status update}

A status update will be presented at the IAU XXVII General Assembly in the Commission 26 Business Meeting in Rio de Janeiro, Brazil, August 2009.

Brian D. Mason \& William I. Hartkopf co-chairs of the Working Group 